\title{
Investigation of the Donation Attitude- Behaviour Gap to Celebrity-Endorsed Charitable Campaigns: An Abstract
}

\author{
Parichehr Riahi Pour
}

\begin{abstract}
The average amount donated to charities has been declining in the recent years in the UK as the pool of donors is decreasing (Chatzidakis et al. 2016). Yet, regardless of the expenses for launching celebrity-endorsed campaigns, charities are increasingly relying on celebrities to promote their causes (Einolf et al. 2013; Littler 2015; Panic et al. 2016). Research about celebrity endorsement in the context of charity campaigns has remained sparse (Keel and Nataraajan 2012; Harris and Ruth 2015; Panic et al. 2016). A pertinent question, therefore, is the role of celebrity endorsers on shaping individuals' donation intentions and behaviour to charities.

Attitudes to donate are widespread in the UK but higher than donation behaviour, as approximately half of individuals with pro-charitable donation attitudes do not donate any money to a charity in a month (Dobbs et al. 2011). This demonstrates that attitudes are not definite proxies for donation behaviour, suggesting that a change in research focus is required.

Conducted and content analysed 17 semi-structured interviews as recommended by Miles and Huberman (1994), this paper introduces new theoretical self-regulatory (Sniehotta et al. 2009) and self-identity congruence (Choi and Rifon 2012) constructs to adapt the theory of planned behaviour (Ajzen and Madden 1986) for understanding the role of celebrity endorsement in reinforcing donation behaviours and intentions. Data were collected until reaching saturation when no new information has emerged (Creswell 2007). Snowball sampling was chosen. The study sought diversity in participants' profiles, essential for tapping into the intricateness of donation attitude-behaviour gap.

The managerial implication is to investigate under what conditions celebrity endorsement is reaping returns for the charities. This insight is crucial for the charities depending on a shrinking pool of donors yet deploying celebrity-endorsed campaigns despite their expenses. This research is currently been developing upon adopting a mixed-methods approach and undertaking questionnaires.
\end{abstract}

P.R. Pour $(\bowtie)$

University of Glasgow, Glasgow, UK

e-mail: p.riahi-pour.1@ research.gla.ac.uk 